\title{
AN IMPROVED EQUATION OF STATE FOR HYDROGEN
}

\author{
D. SAUMON \\ Lunar and Planetary Laboratory, University of Arizona, Tucson AZ 85721
}

G. CHABRIER

Ecole Normale Supérieure, 69364 Lyon Cedex 07, France

An improved theory of fluid hydrogen at high density, based on a detailed treatment of inter-particle correlations and a self-consistent treatment of pressure ionization, has become available recently (Chabrier 1990, Saumon and Chabrier 1991,1992). We present a preliminary comparison between this new EOS (hereatfer SC) and equations of state frequently used in astrophysical contexts, namely: Fontaine, Graboske and Van Horn 1977 (FGVH), Däppen et al. 1988 (MHD) and Magni and Mazzitelli 1979 (MM).

The SC theory predicts a first-order phase transition in the region of pressureionization (the so-called Plasma Phase Transition, or PPT), between an essentially neutral mixture of atoms and molecules $\left(x_{e^{-}}<10^{-2}\right)$, and a partially ionized plasma $\left(x_{e^{-}} \approx 50 \%\right)$, with a critical point located at $P_{c}=0.614 \mathrm{Mbar}$, $T_{c}=15300 \mathrm{~K}$ and $\rho_{c}=0.35 \mathrm{~g} / \mathrm{cm}^{3}$.

We have selected 6 isotherms common to all four EOS, except for the three hottest isotherms which required $T$-interpolation in the MM table. These isotherms are $\log T=3.70,4.10,4.50,5.30,6.10$, and 6.90 . The quantities compared are $\log P$ and $\log U$ for all six isotherms. Exceptions are the MHD EOS which is not plotted for the two hottest isotherms (the table available to us is limited to $\log T \leq 6)$ and the MM EOS which does not provide $\log U$.

All quantities compared here are taken directly from the original EOS tables. In all cases, the zero of energy is chosen as the ground state of the $\mathrm{H}_{2}$ molecule and the contribution of the photon gas is included. Note that the two lowest isotherms cross the PPT of the SC EOS which is represented by a gap near $\log \rho=-0.25$ in the corresponding curves.

As can be seen in the figures, the differences can be so large that it is not necessary to plot ratios or differences between the various EOS! Recall that hydrogen is ideal to better than $1 \%$ for $\log \rho<-2$ (based on the SC EOS). Surprisingly, significant differences are seen even for $\log \rho<-2$. Each quantity is briefly discussed below.

$\log P$ :

MM has a rather high pressure at low- $T$ in the ideal gas regime $(-4<$ $\log \rho<-2)$. This feature is hard to explain. All four equations of state agree very well in the fully ionized regime where the plasma is weakly to moderately coupled and electrons are partially degenerate and begin to dominate 

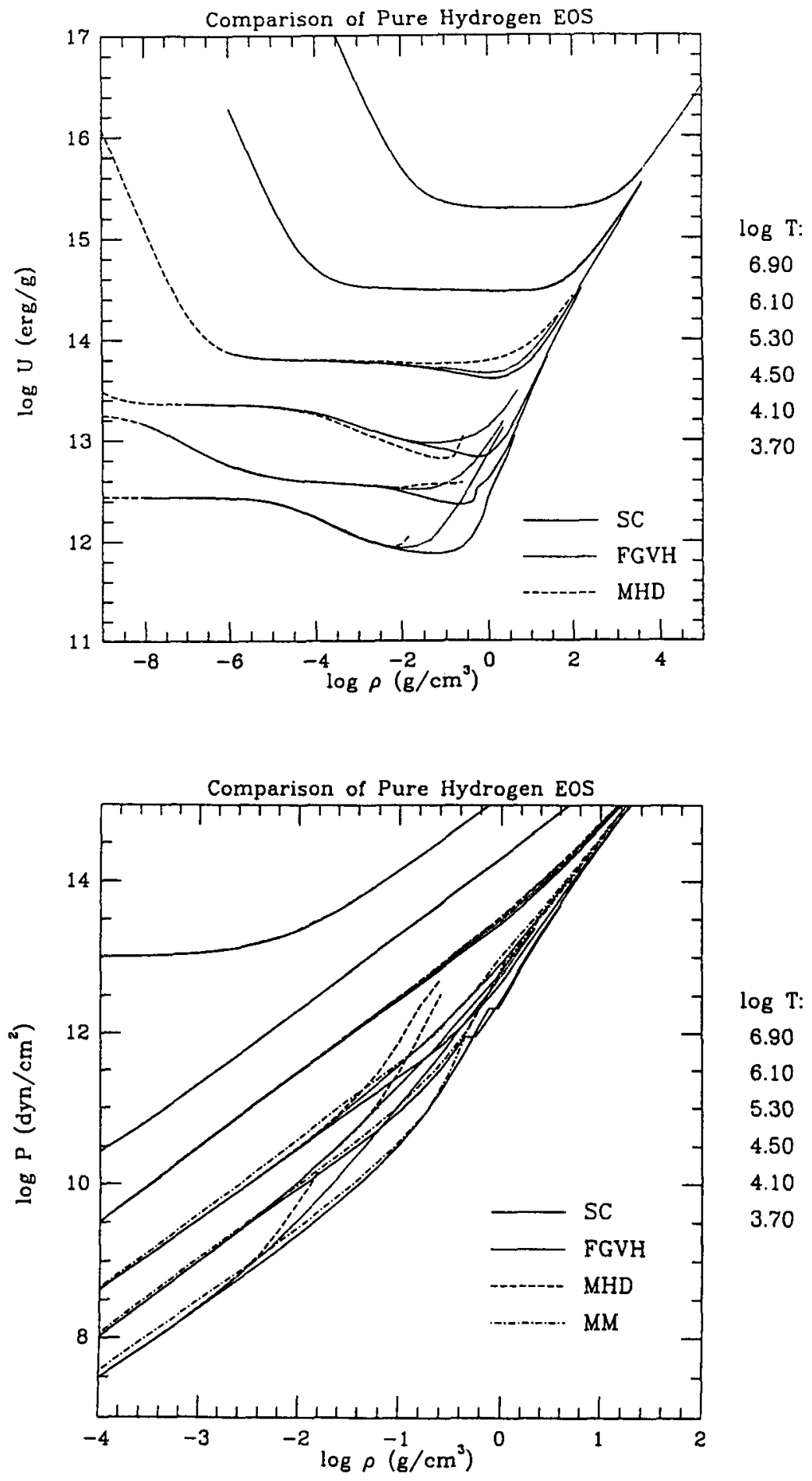
the total pressure. At low- $T$ and at intermediate densities $(\log T \leq 4.50$, $-2<\log \rho<-0.5$ ), MHD and FGVH are up to an order of magnitude above SC. A comparison with the results of shock tube experiments show that the SC EOS is quite good in that regime (Saumon and Chabrier 1991, 1992). FGVH and MHD overestimate the pressure because they use hard sphere interactions between neutral particles which are much too repulsive. Also, MHD uses a rather high value for the hard sphere diameter of $\mathrm{H}_{2}$ (and a small one for $\mathrm{H}$, see Fig. 6 of Saumon and Chabrier 1991), which enhances the effect.

$\log U$ :

Most of the effects discussed above are also visible in the internal energy. The differences between $\mathrm{SC}$ and MHD for $\log T=4.50$ and 5.30 are due to a different treatment of the bound states of $\mathrm{H}$. The MHD theory for the treatment of bound states includes two contributions: a contribution from surrounding neutral particles (excluded volume), and a contribution from surrounding charged particles (Stark ionization). The SC model improves upon MHD for the first contribution (it includes non-linear terms in the hard sphere interactions for the ground state of the particles) but it does not include the second contribution, i.e. the dissolution of the upper levels due to Stark ionization by the microfield of the plasma (see Saumon and Chabrier 1992 for details). The effect of this missing physical effect in SC becomes noticeable when thermal excitation and ionization are significant.

The previous remarks still apply for the second derivatives (not shown here). Substantial disagreement again appears at low to intermediate temperatures in the $-3<\log \rho<1$ domain, where $\nabla_{a d}, \chi_{\rho}$ and $\chi_{T}$ are very sensitive to the interaction models and the treatment of the internal partition functions.

The present hydrogen EOS has been combined with a more simple helium $\mathrm{EOS}$, using the additive volume rule. In a first application of this $\mathrm{H} / \mathrm{He} \mathrm{EOS}$, new static interior models and evolutionary models of Jupiter, Saturn and brown dwarfs were computed. They are presented in Chabrier et al. 1992 and Saumon et al. 1992, where the effect of the PPT on the mechanical and thermal structures of these objects is discussed in details.

\section{REFERENCES}

Chabrier G., J. de Physique, 51, 1607 (1990)

Chabrier G., Saumon D., Hubbard W. B., and Lunine J. I., ApJ, 391, 817, (1992)

Däppen, W., Mihalas, D., Hummer, D. G., and Mihalas, B. W. ApJ, 332, 261 (1988)

Fontaine G., Graboske and Van Horn H. M., ApJS, 35, 293 (1977)

Magni M. and Mazzitelli I., $A \& A, 72,134$ (1979)

Saumon D., Chabrier G., Phys. Rev. A, 44, 5122, (1991); ibid., in press (1992)

Saumon D., Hubbard W. B., Chabrier G., and VanHorn H. M., ApJ, 391, 827 (1992) 